\title{
The Minimum Number of Sensors - Interpolation of Spatial Temperature Profiles in Chilled Transports
}

\author{
Reiner Jedermann and Walter Lang \\ Microsystems Center Bremen (MCB) \\ University of Bremen, Otto Hahn Allee NW1, D-28359 Bremen, Germany \\ rjedermann@imsas. uni-bremen. de
}

\begin{abstract}
Wireless sensor networks are an important tool for the supervision of cool chains. Previous research with a high number of measurement points revealed spatial temperature deviations of more than $5{ }^{\circ} \mathrm{C}$ in chilled transport, but the number of sensors has to be reduced to an economically useful value for use in regular transport. This paper presents a method to estimate the minimum number of sensors and to compare different sensor positioning strategies. Different methods of interpolating the temperature data of intermediate positions were applied to the experimental data from a delivery truck. The average prediction error for intermediate points was estimated as a function of the number of sensors. The Kriging method, originally developed for the interpolation of geostatistical data, produced the best results.
\end{abstract}

Keywords: Wireless sensor networks, Food logistics, Kriging, Information Processing, Temperature mapping.

\section{Wireless Sensors in Cool Chain Management}

Product losses in food transportation due to temperature mismanagement and quality decay can reach up to $35 \%$ [1]. These losses can be mitigated by better supervision of the cool chain. If stock rotation is based on dynamic shelf life or current product quality instead of a fixed production date, quality losses in meat could be reduced from $16 \%$ to $8 \%$, as the group of Taoukis has shown [2], and those in fish could be reduced from $15 \%$ to $5 \%$ [3].

The prediction of shelf life requires temperature monitoring for individual product batches. Temperature differences inside a truck of up to 12 Kelvin [4] can cause severe deviations in product quality. Sea containers with air ducts in the floor allow for a better air flow distribution, but differences between pallet surfaces and core temperatures can still reach 6 Kelvin [5].

Wireless sensor networks provide online access to temperature data during transport. Instant notifications of food quality problems allow for corrective actions to be taken before the transport arrives at its destination. The prediction of shelf life losses can be calculated by an automated system inside the means of transport [6]. Sensors packed inside the cargo are often lost by the end of the transport. In order to avoid 
high sensor replacement costs, it is not feasible to equip each box or pallet inside a truck or container with a sensor node. Instead of oversampling the cargo hold by implementing a high number of measurement points, the temperature has to be interpolated between the positions of a reduced number of sensors. Furthermore, the operating conditions of food transport place high physical demands on the sensors. The sensors must be able to operate in temperatures below $-20{ }^{\circ} \mathrm{C}$, withstand high air humidity and condensation, and endure cleaning by steam jet.

\section{Required Number of Sensors}

The goal of this study is to develop a method to the estimate the number of sensors required to accurately interpolate a spatial temperature profile. The difference between the real temperature and the temperature predicted by interpolation increases for low numbers of source or input sensors. Inappropriate positioning of the input sensors and inaccuracies of the interpolation methods also lead to higher errors.

This paper begins by defining a measure for the interpolation error and introducing the experimental data. The following section compares different interpolation methods for a fixed number of input sensors. A further analysis of the data tests to what extent the interpolation error is reduced by increasing the number of sensors. The effects of different strategies to determine locations for the placement of additional sensors are evaluated. The last section demonstrates how the interpolation error could also be utilized as an indicator of the probability of sensor faults.

\subsection{Source and Destination Points}

The measurement data set was split into two groups. The first group of sensor locations serves as the input for the interpolation model. This group contains the $N_{S}$ source points $s_{i}$. The second group of sensor locations serves as a reference. The measurements at the $N_{Z}$ destination points $z_{i}$ were compared to the output of the selected interpolation model. ${ }^{1}$

\subsection{Definition of Interpolation Error}

The error $\varepsilon_{\mathrm{i}}$ for one destination point $i$ was defined as the median square deviation between the predicted $\bar{z}_{i}$ and the measured $z_{i}$ over $N_{K}$ samples for the transport duration:

$$
\varepsilon_{i}^{2}=\frac{\sum_{k=1}^{N_{K}}\left(\bar{z}_{i}(k)-z_{i}(k)\right)^{2}}{N_{K}}
$$

\footnotetext{
${ }^{1}$ Vectors are marked by bold lowercase letters, matrices by bold capital letters, and transposed matrices by an additional superscript ${ }^{\mathrm{C}} \mathrm{T}$, . Temperature differences are given in Kelvin $[\mathrm{K}]$ and absolute temperatures are given in degrees Celsius $\left[{ }^{\circ} \mathrm{C}\right]$. Averages are indicated by an overbar. Context clarifies whether the average is taken over the transport duration or over all measurement points for a certain sampling instance.
} 
The quality of the interpolation methods was evaluated according to the average prediction error $\bar{\varepsilon}$ over all destination points:

$$
\bar{\varepsilon}=\frac{\sum_{i=1}^{N_{Z}} \varepsilon_{i}}{N_{Z}}
$$

\section{Experimental Data}

With the purpose of conforming to the mechanical requirements of cool chain transport, we equipped TelosB sensors with polyamide water-protected (IP65) housings and external SHT75 temperature and humidity sensors. In order to evaluate the error of the temperature interpolation, it is necessary to acquire data for more points than the final number of sensor positions. Because we have only manufactured a limited number of sensors, we performed the preliminary tests with low-cost data loggers. The tests were performed inside delivery trucks provided by the German company Rungis Express, which specializes in supplying high-quality food products to hotels and restaurants. The cargo hold of each truck is separated into three different temperature zones. During two test transports, 40 TurboTag data loggers were placed in each middle compartment in deep freezer mode with a set point of $-29{ }^{\circ} \mathrm{C}$.

Further details of the test are described in [7], whereas this paper focuses on an analysis of the experimental data to determine the minimum number of sensors and their optimal positions.

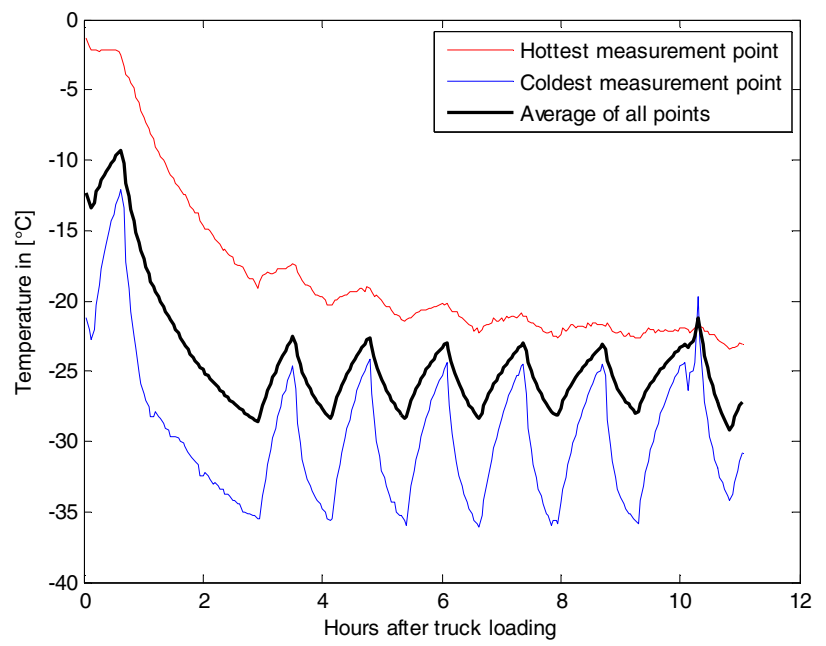

Fig. 1. Temperature over time. Minimum, maximum, and average for transport 1. 


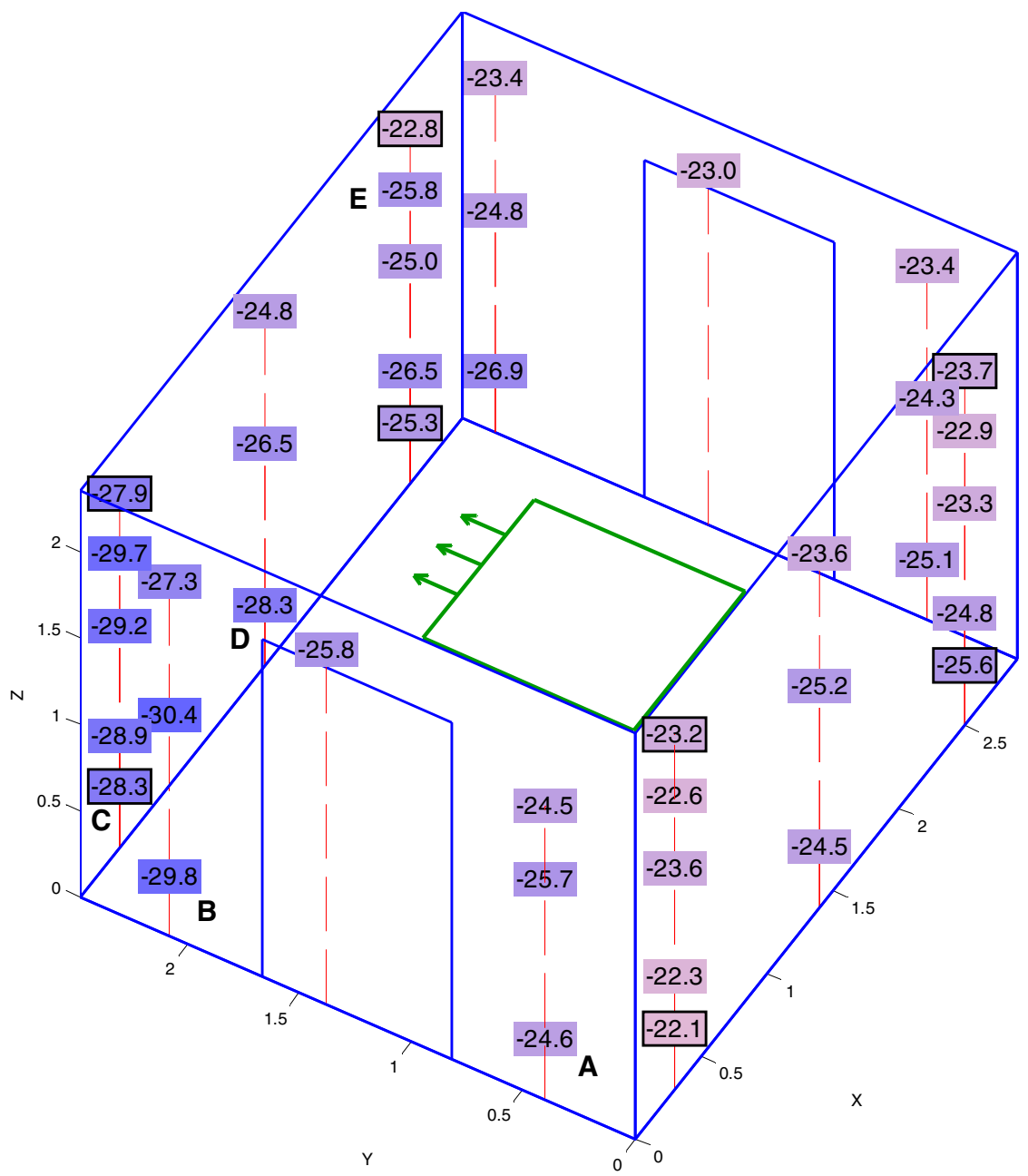

Fig. 2. Spatial temperature distribution at the end of the cooling period in transport 1 . The cooling unit is mounted under the ceiling. The direction of air flow at the ventilation outlet is marked by the three arrows.

Figure 1 shows the temperature over time diagram of both the coldest and the hottest measurement points, as well as the average temperature of all points over the 11hour cooling period during the first transport. The oscillations of the temperature were caused by the on/off cycles of the cooling unit with a period of approximately 1.2 hours. The automated defrosting caused a short temperature peak after 10.5 hours.

The spatial temperature distribution is given in Figure 2. The average over time was calculated for each measurement point over a two hour period starting 8 hours after truck was loaded. Temperature differences of about $7.5 \mathrm{~K}$ are still present at the end of the transport. 


\subsection{Time Correction}

Data loggers are cheap and easy-to-use wireless devices, but they do not provide access to the sensor data during the transport. Furthermore, they do not feature networked time synchronization, which meshed sensor nodes provide. Data loggers can exhibit considerable deviations of the sampling intervals, especially in deep freezer conditions. We found clock deviations of $\pm 3 \%$ in our experimental data. The data was converted to an equal sampling interval of 2.5 minutes by the resample() function in MATLAB. This function resamples the input with a fixed frequency ratio of $p / q$ by upsampling the input by the factor $p$, filtering alias frequencies, and downsampling by the factor $q$. A graphical comparison showed that the error introduced by the resampling was lower than 0.05 Kelvin except for occasional peaks of up to 0.1 Kelvin. Only samples at the beginning and end of the data set, which could have a higher error, were removed before further processing.

\section{Methods for Spatial Interpolation}

The following section compares different approaches for estimating the temperature at the destination points by a linear combination of the values at the source points. The prediction of the destination point $\bar{z}_{i}$ at the sampling instance $k$ is given by the sum of the data of the source points $s_{j}$ multiplied with the time-invariant weighting coefficients $w_{i j}$. The forecast depends solely on the current source values for these linear methods; their prediction model does not contain any state variables.

$$
\bar{z}_{i}(k)=\sum_{j=1}^{N_{S}} s_{j}(k) \cdot w_{i j}
$$

\subsection{Inverse Distance Weighting}

Inverse distance weighting is most common method for determining the weighting coefficients. This method uses only the geometrical distances between the source points. It assumes that the influence of a source point $s_{j}$ on a destination point $z_{i}$ decreases with the square of their distance $h_{i j}$. The weighting coefficients are given by equation (4). The additional parameters $\omega_{i}$ are used to scale the weighting coefficients in such a way that their sum equals 1 for each destination point.

$$
w_{i j}=\frac{\omega_{i}}{h_{i j}^{2}} \text { with } \omega_{i}=\left(\sum_{i=1}^{N_{Z}} \frac{1}{h_{i j}^{2}}\right)^{-1}
$$

\subsection{Kriging}

An improved interpolation method was developed by D.G. Krige [8] in the 1950s for the exploration of mineral resources with 1000 or more test drillings. To date, 
the Kriging method has only been scarcely utilized in sensor networks; see [9] for example. This might be due to the fact that, in general, there are a lower number of probe points in wireless sensor applications than in geological research. We tested whether Kriging could also calculate a precise prediction for small data sets with only 40 points of experimental data. The Kriging method, as described in equation (5)-(8) [10], [11], was implemented as a set of MATLAB functions. As new applications of Kriging we used this method to estimate the number of required sensors and to test the plausibility of the sensor data.

Kriging calculates the solution with the least possible expected value for the errors $\varepsilon_{i}$ of each destination point by assuming the following:

a) The mean of the measurement values is independent of space, and

b) The expected value for the temperature difference between two points depends solely on their spatial distance vector.

Depending on the data set, in many cases it is possible to reduce the second assumption to an isotropic form in which the difference does not depend on the direction but only on the absolute value of the distance.

Kriging can be seen as an improved form of inverse distance weighting. Whereas the inverse distance method calculates the weighting coefficients directly by the geometrical distance between two points $h_{i j}$, Kriging uses the variogram $v\left(h_{i j}\right)$ that expresses the statistical dependency of two points as function of their distance $h_{i j}$. The variogram describes the statistical dependency by the expected value $E$ for the square of the temperature difference of two points $i, j$ :

$$
v\left(h_{i j}\right)=\frac{1}{2} E\left\{\left(s_{i}(k)-s_{j}(k)\right)^{2}\right\}
$$

The primary disadvantage of the Kriging method lies in its estimation of the variogram from the measurement values. Because our data set with 40 sensor locations did not allow for a determination of separate variograms for different directions of the distance vector, an isotropic distribution was assumed. In contrast to geological research in which only one static value per probe point is taken, we obtained a time-variant series of measurements. The equation to estimate the experimental variogram $v^{*}(h)$ from the data was slightly modified, resulting in the following:

$$
v_{i, j}^{*}\left(h_{i, j}\right)=\frac{1}{2} \cdot \sum_{k=1}^{N_{K}}\left(s_{i}(k)-s_{j}(k)\right)^{2}
$$

The value was calculated for the first transport with all $0.5 \cdot 40 \cdot(40-1)=780$ possible combinations of two points. The resulting values for $v^{*}(h)$ were grouped by the absolute value of the distance in intervals of 0.25 meter length. The average value for $v^{*}$ (h) inside each interval is given by a marker in Figure 3. 


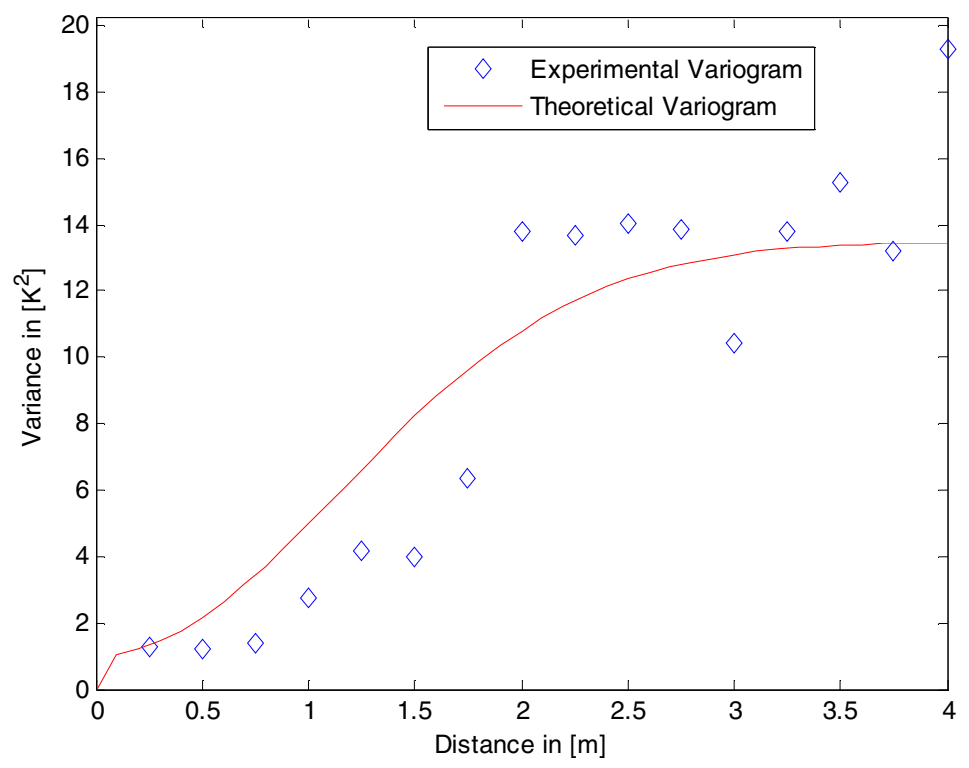

Fig. 3. Experimental and theoretical variogram

This experimental curve was approximated by a theoretical variogram $v(h)$. Only a limited set of functions can be applied as theoretical variograms. The function must conform to several limitations. For example, the variogram has to be a monotonically increasing function. In addition, apart from the origin, the function has to be continuous. $^{2}$ The Gaussian model was selected from the supposed standard models [10], [11] because it produced the best fit for the gentle rise of the curve for small distances:

$$
v(h)=v_{0}+\left(v_{\infty}-v_{0}\right) \cdot\left(1-e^{-(3 h / r)^{2}}\right)
$$

The radius $r$ as the primary parameter of the variogram can be interpreted as the maximum distance for the mutual influence between two points. The initial value $v_{0}$ is the minimum value of variance for distances greater than zero. $v_{\infty}$ gives the variance for large distances. The parameters of the variogram for the data from the first transport were estimated to be $r=2.8 \mathrm{~m}, v_{0}=1.0 \mathrm{~K}^{2}, \mathrm{v}_{\infty}=13.5 \mathrm{~K}^{2}$.

Analyses of the data from the second transport showed higher initial and final values $\left(v_{0}=2.5 \mathrm{~K}^{2}, \quad \mathrm{v}_{\infty}=32.5 \mathrm{~K}^{2}\right)$, but no significant difference in the radius.

The method of inverse distance weighting directly calculates the weighting coefficients as a function of distance, whereas the Kriging method also considers the mutual influence of all measurement points by a linear system of equations. The Ordinary Kriging method also estimates the spatial average $\mu$ as an auxiliary variable:

${ }^{2}$ Only functions that are negative semi-definite can be utilized as variogram [11]. Otherwise, equation (12) on page 12 could result in a negative value for the Kriging variance. 


$$
\left[\begin{array}{cccc}
v_{1,1} & & v_{1, N_{S}} & 1 \\
\vdots & \ddots & \vdots & \vdots \\
v_{N_{S}, 1} & \cdots & v_{N_{S}, N_{S}} & 1 \\
1 & \cdots & 1 & 0
\end{array}\right] \cdot\left[\begin{array}{c}
w_{1, q} \\
\vdots \\
w_{N_{S}, q} \\
\mu
\end{array}\right]=\left[\begin{array}{c}
v_{1, q} \\
\vdots \\
v_{N_{S}, q} \\
1
\end{array}\right]
$$

The matrix on the left side contains the values of the variogram for the distances between all source points $v_{i, j}$. The vector on the right side contains the values $v_{i, q}$ for the distances between each source point and one destination point $z_{q}$. The weighting coefficients $w_{i, q}$ used in calculating the destination point are retrieved by solving the linear set of equations in (8).

\subsection{Kriging with Spatial Trends}

The Ordinary Kriging method assumes that the mean value of the temperature is constant over space, but our experimental data showed a difference of $4 \mathrm{~K}$ between the average temperature of the left and right walls. There are three methods in which this problem can be addressed.

The first method is to simply ignore the trend and directly apply Ordinary Kriging, assuming that the effect of the trend is negligible for small distances.

The second method implements the following steps: a) A linear or polynomial trend model is estimated from the source points. b) The prediction of the trend model is subtracted from the source points. c) Ordinary Kriging is applied to the difference. d) The trend model is added to the destination points obtained by Ordinary Kriging.

The third approach using the Universal Kriging method extends the set of linear equations in (8). The parameter values of a trend model are calculated as additional variables. However, Universal Kriging is only necessary if the temperature distribution exhibits local drifts in addition to a global trend [10, page 38].

Because our data set of 40 measurement points does not contain enough information to estimate local drifts or cubic trend functions, only the second approach involving a linear model was applied. The temperature trend $T^{*}$ was predicted as a function of the coordinates $p_{x}, p_{y}, p_{z}$, of a point $i$. The model parameters $\alpha$ were estimated separately for each sampling instance $k$ :

$$
T^{*}(i, k)=\alpha_{0}(k)+\alpha_{1}(k) \cdot p_{x}(i)+\alpha_{2}(k) \cdot p_{y}(i)+\alpha_{3}(k) \cdot p_{z}(i)
$$

For the first transport, the average over time of the parameters was calculated as $\bar{\alpha}_{0}=-21.98^{\circ} \mathrm{C}, \quad \bar{\alpha}_{1}=0.17 \mathrm{~K} / \mathrm{m}, \quad \bar{\alpha}_{2}=-1.98 \mathrm{~K} / \mathrm{m}, \quad \bar{\alpha}_{3}=0.15 \mathrm{~K} / \mathrm{m}$.

\section{Comparison of Interpolation Errors}

The described interpolation methods were applied to the recorded data of both transports. We evaluated the data set twice, once using 8 source points and once using 30 source points. From the 40 total points, either 32 or 10 remained as destination points. The average interpolation error was calculated for these remaining points. The set with 8 source points includes the positions in the corners of the cargo hold that are 
marked by a black frame in Figure 2. The positions of the sensors for the set with 30 source points were selected according to the approach described in the next section.

Two further simple interpolation approaches were applied as references for the comparison: a) The time-dependent average of the source points was taken to predict all destination points, independent of their locations. b) The destination values were set according to the trend model in equation (9). The results of the comparison are summarized in Table 1:

Table 1. Comparison of interpolation error $\bar{\varepsilon}$ for different methods for 8 and 30 source points

\begin{tabular}{lllll}
\hline Experiment / Source points & Ex1/8 & Ex2/8 & Ex1/30 & Ex2/30 \\
\hline Average of source points & $2.796 \mathrm{~K}$ & $3.912 \mathrm{~K}$ & $2.567 \mathrm{~K}$ & $3.239 \mathrm{~K}$ \\
Linear trend & $1.984 \mathrm{~K}$ & $2.723 \mathrm{~K}$ & $1.881 \mathrm{~K}$ & $3.437 \mathrm{~K}$ \\
Inverse distance weighting & $1.443 \mathrm{~K}$ & $2.287 \mathrm{~K}$ & $1.105 \mathrm{~K}$ & $1.720 \mathrm{~K}$ \\
Ordinary Kriging & $1.389 \mathrm{~K}$ & $2.170 \mathrm{~K}$ & $0.530 \mathrm{~K}$ & $1.325 \mathrm{~K}$ \\
Kriging with linear trend & $1.418 \mathrm{~K}$ & $2.231 \mathrm{~K}$ & $0.533 \mathrm{~K}$ & $1.474 \mathrm{~K}$ \\
\hline
\end{tabular}

Ordinary Kriging gave the most accurate prediction with an interpolation error between $0.5 \mathrm{~K}$ and $2.2 \mathrm{~K}$. The combination of Kriging with a linear trend model resulted in a slightly higher error. A linear trend, which affects the whole cargo hold, fails to properly explain the difference between the average temperatures of the left and right walls. Thus, for this data set, the best approach is to directly apply Ordinary Kriging, which gives the best fit for randomly distributed heat sources.

The accuracy of Kriging increases with the number of source points. A comparison of the interpolation error of Ordinary Kriging with other methods gave the following results: For 30 source points, the error is reduced by $52 \%$ for the first and $23 \%$ for the second transport compared to inverse distance weighting. Compared to the simple average and the linear trend model, the improvement is between 59\% and $79 \%$.

The variograms for the first and second transports exhibited differences only in their initial and final values, with a similar relation of $v_{\infty} / v_{0}$. The variogram for the second experiment can be approximated by a proportional scaling of the first variogram:

$$
v_{\mathrm{sec} \text { ond }}(h) \approx 2.4 \cdot v_{\text {first }}(h)
$$

The factor 2.4 can be reduced in equation (8) because it appears on both sides. Therefore, the weighting matrix for Kriging is almost independent of the number of the experiment. The effect of the differences in the variogram was calculated to be lower than $0.1 \%$.

\subsection{Comparison with Linear Curve Fitting}

The coefficients of the weighting matrix depend mainly on the geometrical locations of the measurement points for the inverse distance and the Kriging method. The weighting coefficients are almost independent of the current sensor data for these two methods. 
Other modeling approaches set the weighing coefficients in order to give the best fit for a set of training data. A disadvantage of these approaches is that in addition to the values of the source points, the values of the destination points must also be known in advance for use as training data. Because the weighing coefficients depend on the sensor data of the training experiment, it is necessary to test by a crossvalidation whether it is possible to apply the model to future experiments. Linear curve fitting is introduced as an example for this approach.

Equation (3) was transferred to a matrix form. The rows of the matrices $\hat{\mathbf{Z}}$ and $\mathbf{S}$ contain all measurement values at one sampling instance. The matrix $\mathbf{W}$ contains the time-invariant weighting coefficients:

$$
\widehat{\mathbf{Z}}=\mathbf{S} \cdot \mathbf{W}
$$

The weighting coefficients are calculated as the least square error solution of the overdetermined linear set of equations (10) by the Moore-Penrose pseudoinverse [12]:

$$
\mathbf{W}=\left(\mathbf{S} \cdot \mathbf{S}^{\mathbf{T}}\right)^{-1} \cdot \mathbf{S}^{\mathbf{T}} \cdot \mathbf{Z}
$$

If the weighting matrix is recalculated by equation (11) for each experiment, the curve fitting delivers a superb approximation that is between $75 \%$ and $87 \%$ better than those calculated with Kriging (Table 2). However, the curve fitting fails to give a prediction for experiments in which the destination values are not known in advance. The weighting matrix was calculated with the data of experiment one and applied to experiment two and vice versa. The interpolation error of this cross-validation was always larger than the error predicted by Kriging. The prediction for the destination points in experiment one was hardly better than the simple average of source values.

Table 2. Interpolation error $\bar{\varepsilon}$ and cross validation for linear curve fitting

\begin{tabular}{lllll}
\hline Experiment / Source points & Ex1/8 & Ex2 / 8 & Ex1 / 30 & Ex2 / 30 \\
\hline Linear curve fitting & $0.283 \mathrm{~K}$ & $0.415 \mathrm{~K}$ & $0.134 \mathrm{~K}$ & $0.177 \mathrm{~K}$ \\
Cross validation curve fitting & $2.638 \mathrm{~K}$ & $2.234 \mathrm{~K}$ & $2.417 \mathrm{~K}$ & $1.470 \mathrm{~K}$ \\
\hline
\end{tabular}

This problem might also appear in other learning methods in which the parameters of the prediction system are trained by the data of one or more experiments. Their ability to predict destination values in future experiments has to be tested by careful cross-validation.

\section{The Number of Required Sensors}

The prediction error of the Kriging method decreases with the number of source points as shown in Table 1. In order to answer the initial question of this paper concerning the required number of sensors, the average prediction error of the Ordinary Kriging method was plotted in Figure 4 as a function of the number of source points. Starting with a configuration of one sensor in each of the eight corners, new sensors were added one by one. Furthermore, the effect of the location of the new sensors on 


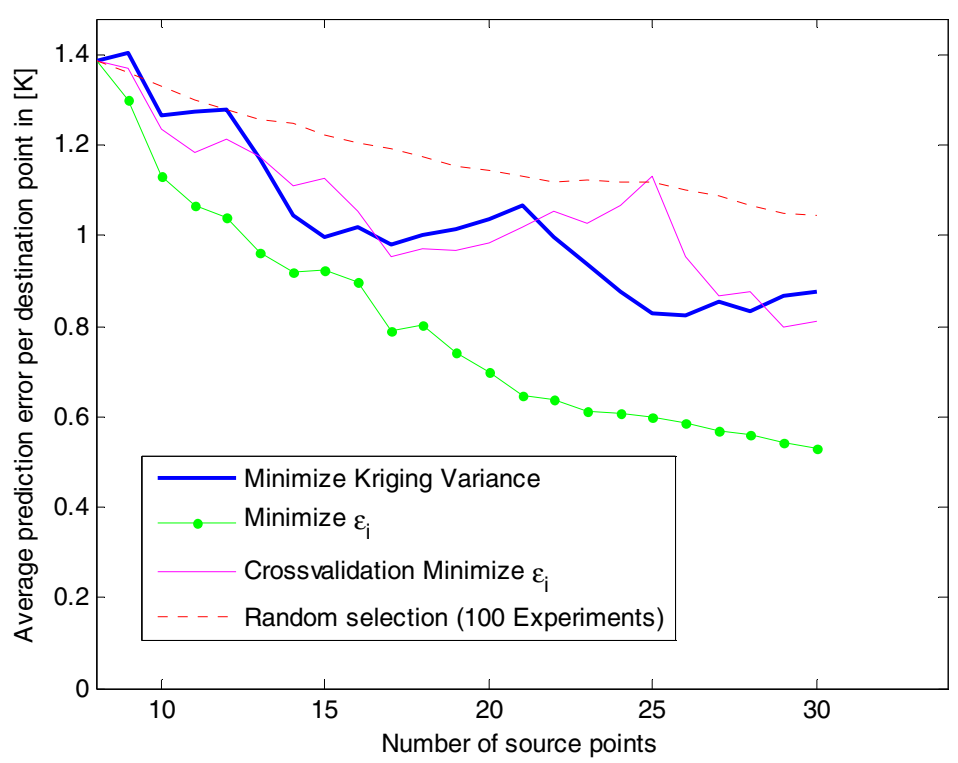

Fig. 4. Average prediction error $\bar{\varepsilon}$ as a function of the number of source points for different sensor-addition strategies

the prediction error was tested by this simulation. New points were added according to four different strategies:

a) New points were added randomly. The curve shows the average of 100 random experiments.

b) The Kriging method also provides the means to estimate the expected error in unknown destination points. The Kriging variance KV or its square root, the Kriging standard deviation $\sigma_{K}$, can be calculated as the product of the two vectors in equation (8):

$$
K V=\sigma_{K}^{2}=\left[\begin{array}{c}
w_{1, q} \\
\vdots \\
w_{N_{S}, q} \\
\mu
\end{array}\right]^{T} \cdot\left[\begin{array}{c}
v_{1, q} \\
\vdots \\
v_{N_{S}, q} \\
1
\end{array}\right]
$$

The goal of this strategy b) is to minimize the average Kriging variance of the remaining destination points. All options to convert one destination point into a source point were tested. The point that resulted in the lowest average was selected as new source point.

c) The destination point with the highest deviation between the predicted and measured $\varepsilon_{i}$ was converted into a source point.

d) Whereas the weighting coefficients are independent of the number of the experiment, the cold and hot spots as locations of temperature extremes can change location for different transports. Thus, the points with the 
maximum $\varepsilon_{i}$ are also subject to change. Therefore, strategy c) has to be tested by cross-validation. The sequence for adding new sensors was determined according to the measurements from experiment two by employing strategy c). Thereafter, the same sequence was applied to experiment one.

A share of the measured prediction errors $\varepsilon_{i}$ results from calibration tolerances of the sensors at the destination points. The average measurement error was estimated by a test in a climatic chamber for a set of 36 TurboTag data loggers. Their temperature measurement exhibited a standard deviation of $0.25 \mathrm{~K}$ at $0{ }^{\circ} \mathrm{C}, 0.38 \mathrm{~K}$ at $-10{ }^{\circ} \mathrm{C}$, and $0.68 \mathrm{~K}$ at $-25{ }^{\circ} \mathrm{C}$. The average temperature in our experiments was approximately $25^{\circ} \mathrm{C}$. Therefore, the related tolerance of $0.68 \mathrm{~K}$ should be considered as the low boundary for the prediction error.

In general, not only the temperatures at the remaining destination points of the data set should be estimated, but also those at any point inside the cargo hold. Therefore, the simulation was stopped after 30 source points were added. Otherwise, there would not have been a sufficient number of destination points remaining to reliably calculate the average prediction error.

Only strategy c) goes slightly below the low boundary, because it selects the points directly according to their prediction error, thus disregarding sensors with high tolerances. The other three strategies showed a slower decrease of the prediction error. If new points are added by one of these strategies, far more than 30 source points are necessary to reach the low boundary.

The four strategies were compared on the basis of the average error $\varepsilon_{20-30}$ over the interval between 20 and 30 source points. Strategy c), in which new sensors are added according to the maximum $\varepsilon_{i}$, showed the best result with $\varepsilon_{20-30}=0.60 \mathrm{~K}$. The crossvalidation increased its error to $\varepsilon_{20-30}=0.96 \mathrm{~K}$, which is slightly higher than the error that results from adding new sensors based on the Kriging variance with $\varepsilon_{20-30}=0.91$ $\mathrm{K}$. The improvement using the latter two strategies is less than $20 \%$ compared to the average of the random experiments with $\varepsilon_{20-30}=1.11 \mathrm{~K}$.

In typical applications, little or no sensor data is available before installation. In these cases, it is only possible to determine the sensor positions based on the Kriging variance. Strategy c) can only be applied if a larger data set is available. The number of measurement points in the data set must exceed the number of the reduced sensor positions. The error of strategy c) might increase for data from untrained transports, but this error is only slightly larger compared to errors using other strategies.

Strategies a) to d) are all greedy in the sense that they seek only the instant advantage and cannot change or undo earlier decisions. A fifth algorithm was tested as an example of a less greedy variant of strategy b). The algorithm searches for the best combination of the following three steps in order to minimize the average Kriging variance for the remaining destination points: i) remove one source point; ii) replace it with another point; iii) add a further point as source point. But the advantage of this strategy compared to b) was rather marginal. For 30 points, both strategies resulted in the same list but ordered differently.

The number of sensors required to achieve a given limit for the average prediction error can be obtained from Figure 4. If, for example, the limit is $1.0 \mathrm{~K}$ and the positions are determined by the Kriging variance, at least 22 sensors are necessary. 


\section{Plausibility Testing Based on the Kriging Variance}

The Kriging variance also provides a means of testing the plausibility of single measurements or of the interpolation process itself. This related type of cross-validation selects all measurements as source points except for one. The Kriging standard deviation $\sigma_{K}(i)$ and the prediction error $\varepsilon_{i}$ are calculated for this destination point $i$. The process is repeated after selecting the next location as destination point. Figure 5 shows the resulting values for all destination points.

If the data set satisfies the statistical assumptions of the Kriging method, the average of $\sigma_{K}$ should be equal to the average prediction error. A large difference between these two values indicates that either the expected value of the measurements exhibits a spatial dependency, that a spatial trend or an anisotropic relation of the variogram has not been considered, or that the variogram does not correctly represent the measurements.

The values of the Kriging standard deviation are slightly too high for our data set compared to the measured prediction error. This could be caused by the temperature trend that was observed in direction of the y-axis. If a trend exists which is not compensated in the Kriging process, the variogram can produce excessively high values according to Schafmeister [10, page 37].

The described cross-validation could also test whether the measured values of one or more sensors are plausible. Figure 5 shows four points in which the measured values differ by more than $2 \mathrm{~K}$ from the calculated prediction. These deviations can arise due to the following:

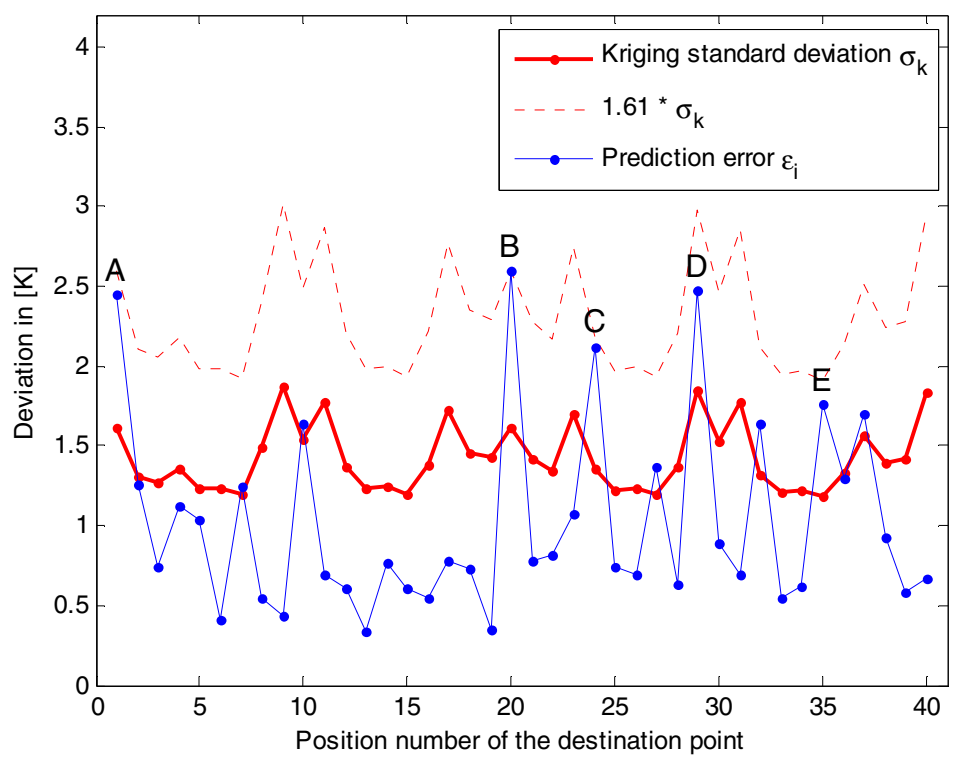

Fig. 5. Kriging standard deviation and prediction error as a function of the position of one destination point 
a) The prediction itself has a high level of uncertainty, because the neighbor source points are too far away. This is expressed by the value of $\sigma_{K}(i)$.

b) The error is caused by random noise or statistical effects.

c) The prediction error has a physical cause. The point could be warmed up by a heat source that produces only a localized effect.

d) The sensor is faulty or exhibits an excessive tolerance.

In order to eliminate option a), the relation between the prediction error and the Kriging standard deviation was used as an indicator of the likelihood of a sensor fault at this point.

$$
F(i)=\frac{\varepsilon_{i}}{\sigma_{K}(i)}
$$

The five points with the highest indicator values $1.3<F(i)<1.61$ are marked with the letters A-E in figures 2 and 5. Point $\mathrm{D}$ has a higher prediction error $\varepsilon_{i}$ than point $\mathrm{E}$, although point $\mathrm{E}$ has a higher indicator value. The error at point $\mathrm{E}$ has to be considered as more significant. The distances to the two next neighbors are only half as great as the distances for point $\mathrm{D}$. Therefore, the prediction at point $\mathrm{E}$ is expected to be more accurate.

Random noise might be the best explanation for the high indicator values of these points. If a Gaussian distribution is assumed, $81 \%$ of the values should be inside the interval $\pm 1.3 \cdot \sigma$. A share of 5 out of 40 sensors with $\mathrm{F}(\mathrm{i})>1.3$ can be explained by the statistical distribution, but other possible explanations for the high prediction errors should also be considered. Points A through D are all located close to the floor of the cargo hold. Presumably, the sensors are blocked by boxes in front of them. These sensors measure the box temperature rather than the distribution of air temperature.

The sensors that are most likely to be faulty can be identified by calculation the Kriging variance in order to check their plausibility. Sensors with a deviation much higher than the Kriging standard deviation should be carefully checked. In our data set, the deviations could be attributed to a physical cause or to noise effects. Except for these cases, sensors with high deviations should be regarded as faulty.

\section{Summary and Conclusion}

Wireless sensor networks can be used to detect local temperature deviations in cool chain transports, but oversampling of the cargo hold by implementing too many sensors should be avoided. The temperature value at any position can be estimated by interpolating the values of a limited number of sensors.

The Kriging method proved to be a useful tool for the evaluation of spatial sensor measurements. It delivers a more accurate interpolation than the commonly used inverse distance weighting. The expected prediction error at positions where no sensor is present can be calculated by using the Kriging variance. The minimum number of sensors can be estimated by a plot of the prediction error as a function of the number of measurement points. The plausibility checking based on the Kriging variance also provides a way to detect faulty sensors. 
The disadvantage of Kriging is that it requires at least one data set with a high number of sensor positions to estimate the required variogram. The 40 positions of our data set seem to suffice for this process only by a small margin.

Although Kriging was originally developed for data sets made up of thousands of positions, the comparison of different interpolation methods showed that Kriging can also be usefully applied to typical sensor networks applications employing lower numbers of measurement points.

\section{Acknowledgment}

This research was supported by the German Research Foundation (DFG) as part of the Collaborative Research Centre 637 "Autonomous Cooperating Logistic Processes". We further thank Rungis Express AG, Germany for the provision of test facilities. See www.intelligentcontainer.com for additional project information.

\section{References}

1. Scheer, P.P.: Optimising supply chains using traceability systems. In: Smith, I., Furness, A. (eds.) Improving traceability in food processing and distribution, pp. 52-64. Woodhead publishing limited Cambridge, England (2006)

2. Koutsoumani, K., Taoukis, P.S., Nychas, G.J.E.: Development of a safety monitoring and assurance system for chilled food products. International Journal of Food microbiology 100(1-3), 253-260 (2005)

3. Tsironi, T., Gogou, E., Taoukis, P.: Chill chain management and shelf life optimization of MAP seabream fillets: A TTI based alternative to FIFO. In: Kreyenschmidt, J. (ed.) 3rd International Workshop on Coldchain Management, Bonn, pp. 83-89 (2008), http: / /www. ccm. uni-bonn. de

4. Moureh, J., Flick, D.: Airflow pattern and temperature distribution in a typical refrigerated truck configuration loaded with pallets. International Journal of Refrigeration 27(5), 464-474 (2004)

5. Tanner, D.J., Amos, N.D.: Heat and Mass Transfer - Temperature Variability during Shipment of Fresh Produce. Acta Horticulturae 599, 193-204 (2003)

6. Jedermann, R., Schouten, R., Sklorz, A., Lang, W., van Kooten, O.: Linking keeping quality models and sensor systems to an autonomous transport supervision system. In: Kreyenschmidt, J., Petersen, B. (eds.) Proceedings of the 2nd international Workshop on Cold Chain-Management, pp. 3-18. University Bonn, Bonn (2006)

7. Jedermann, R., Lang, W.: Semi-passive RFID and beyond: steps towards automated quality tracing in the food chain. International Journal of Radio Frequency Identification Technology and Applications (IJRFITA) 1(3), 247-259 (2007)

8. Krige, D.G.: A statistical approach to some mine valuations and allied problems at the Witwatersrand, Master's thesis of the University of Witwatersrand (1951)

9. Camilli, A., Cugnasca, C.E., Saraiva, A.M., Hirakawa, A.R., Corrêa, P.L.P.: From wireless sensors to field mapping: Anatomy of an application for precision agriculture. Computers and Electronics in Agriculture 58(1), 25-36 (2007)

10. Schafmeister, M.T.: Geostatistik für die hydrogeologische Praxis. Springer, Berlin (1999)

11. Chilès, J.P., Delfiner, P.: Geostatistics - modeling spatial uncertainty. John Wiley \& Sons, New York (1999)

12. Ben-Israel, A., Greville, T.N.E.: Generalized Inverses. Springer, New York (2003) 